\title{
Multimodality standard of care treatment of resectable and borderline resectable pancreatic cancer
}

\author{
John P. Neoptolemos ${ }^{1}$, Paula Ghaneh ${ }^{2}$, Thilo Hackert ${ }^{1}$ \\ ${ }^{1}$ Department of General, Visceral and Transplantation Surgery, University of Heidelberg, Heidelberg, Germany; ${ }^{2}$ Department of Clinical Cancer \\ Medicine, Institute of Systems, Molecular and Integrative Biology, The University of Liverpool, Liverpool, UK \\ Correspondence to: Prof. Dr. med. John P. Neoptolemos, MA, MB, BChir, MD, FRCS, FMedSci, MAE. Professor of Surgery. Department of General, \\ Visceral and Transplantation Surgery, University of Heidelberg, Im Neuenheimer Feld 420, 69120 Heidelberg, Baden-Württemberg, Germany. \\ Email: john.neoptolemos@med.uni-heidelberg.de. \\ Comment on: Kizy S, Altman AM, Wirth KM, et al. Systemic therapy without radiation may be appropriate as neoadjuvant therapy for localized \\ pancreas cancer. Hepatobiliary Surg Nutr 2020;9:296-303.
}

Submitted Mar 27, 2021. Accepted for publication Apr 15, 2021.

doi: 10.21037/hbsn-21-131

View this article at: https://dx.doi.org/10.21037/hbsn-21-131

This retrospective single centre report of 36 patients with resectable pancreatic cancer who had neoadjuvant systemic chemotherapy, found that only 25 (69\%) of patients could undergo resection with a median overall survival of 34.4 months (1). They concluded that a short course of neoadjuvant chemotherapy without chemoradiation may improve patient selection prior to surgical resection (1). Whilst the notion that effective neoadjuvant therapy does not require chemoradiation is interesting this study demonstrates the weaknesses of retrospective studies. Progress in the treatment of pancreatic cancer has only taken place through well designed prospective randomized controlled trials.

Kizy et al. used neoadjuvant chemotherapy in patients with resectable disease but this is without a strong scientific basis (1). Randomized controlled trials have established that the standard of care in resectable disease is adjuvant systematic combination chemotherapy. This has resulted in an amazing increase in 5-year survival from $8 \%$ with resection alone to $30-50 \%$ when this was followed by 6 months combination chemotherapy (2-4). The use of adjuvant chemoradiotherapy in addition to systemic chemotherapy, adds to toxicity and does not improve survival-indeed it may detract from survival (2). The longest overall survival achieved with adjuvant chemotherapy is using modified folinic acid, 5-fluorouracil
(5-FU), irinotecan and oxaliplatin (mFOLFIRINOX), but this comes with added toxicity and frequent hospitalizations compared to adjuvant gemcitabine and capecitabine $(3,4)$. Although mFOLFIRINOX is recommended as the preferred treatment it should be borne in mind that the PRODIGE24 trial used highly selected patients with a more favorable prognosis than those in the ESPAC-4 trial and there may be differences between the two regimens in survival responses in different subgroups $(3,4)$.

The use of neoadjuvant therapy appears to increase resectability and hence survival in patients with otherwise unresectable local disease due to local vessel encasement, even in the presence of resectable oligometastatic disease. An argument has also been made to extend the use of neoadjuvant therapy to patients with resectable disease, because the total adjuvant chemotherapy delivered might be reduced due to post-operative complications (1). The authors state that previous studies in the USA have shown around $60 \%$ of patients complete recommended adjuvant therapy mainly because of complications. This is more likely to be explained by variations in clinical practice rather than any deficiency in the treatment modality. Extending the adjuvant window to 12 weeks after resection to allow for postoperative complications does not affect long term survival $(2,3)$. The most important factor in survival for pancreatic cancer is resection of the primary cancer (3).

$\wedge$ ORCID: 0000-0002-6201-7399. 
In the study by Kizy et al. $31 \%$ of resectable patients were not resected and so contributed to a deleterious survival outcome in this group of patients.

A recent prospective trial of perioperative chemotherapy in patients with resectable pancreatic cancer comprising 12 weeks neoadjuvant chemotherapy, followed by surgery, and then 12 weeks adjuvant chemotherapy were also randomized to either mFOLFIRINOX or gemcitabine plus nab-paclitaxel (5). Two-year overall survival rates (95\% CI) were $47 \%(31-61 \%)$ for mFOLFIRINOX and $48 \%(31-63 \%)$ for gemcitabine plus nab-paclitaxel with median overall survival rates of 23.2 (17.6-45.9) months and 23.6 (17.8-31.7) months respectively. Neither arm had a significantly higher 2-year overall estimate compared to the a priori threshold of $40 \%$ (5). The authors concluded that this phase II randomized clinical trial did not demonstrate an improved overall with neoadjuvant chemotherapy, compared with historical data from adjuvant trials in resectable pancreatic cancer (2-5). Moreover, the overall tumor response rates showed no statistically significant differences which were found in $5(9 \%)$ of 55 patients given mFOLFIRINOX and in $10(21 \%)$ of 47 given gemcitabine with nab-paclitaxel (5).

Randomized studies using a mixture of resectable, borderline resectable and locally advanced disease have tended to produce a confused picture. Two recent trials focusing only on borderline resectable disease, however, have a produced more consistent conclusion $(6,7)$. The ESPAC-5F trial has shown that using neoadjuvant therapy was superior to upfront surgery (6). In this trial 90 patients with NCCN defined borderline resectable pancreatic cancer were randomized to receive immediate surgery $(n=32)$, or neoadjuvant therapy $(n=56)$ of either two cycles of gemcitabine with capecitabine $(n=20)$, or four cycles of FOLFIRINOX ( $\mathrm{n}=20)$, or 50.4 Gy of capecitabinebased chemoradiotherapy $(n=16)(6)$. The resection rate was $62 \%$ for immediate surgery and $55 \%$ for neoadjuvant therapy $(\mathrm{P}=0.67)$ with $\mathrm{R} 0$ resection rates of $15 \%$ and $23 \%$ respectively $(\mathrm{P}=0.72)$. One year survival $(95 \% \mathrm{CI})$ rates were $40 \%$ (26-62\%) for immediate surgery and $77 \%$ (66-89\%) for neoadjuvant therapy $(\mathrm{P}<0.001)$. There was little difference in survival rates between FOLFIRINOX and gemcitabine and both showed better survival than chemoradiotherapy although the numbers were relatively small (6).

In the Alliance A021501 trial 110 patients with borderline resectable pancreatic cancer were randomized to either arm eight cycles of neoadjuvant mFOLFIRINOX, or to seven cycles of mFOLFIRINOX followed by stereotactic body radiotherapy (with 5 fractions of either 33-40 Gy of stereotactic body radiation or 25 Gy hypofractionated image guided radiotherapy followed by four cycles of adjuvant mFOLFOX6 after resection (7). The resection rates were $49 \%$ with mFOLFIRINOX and $35 \%$ for mFOLFIRINOX plus chemoradiotherapy and the median (95\% CI) overall survival was $31.0(22.2-\mathrm{NE})$ months and $17.1(12.8-24.4)$ months respectively (7). Thus, both ESPAC-5F and the Alliance A021501 randomized trials showed that chemoradiotherapy (as in the advanced and adjuvant settings) was inferior to chemotherapy alone $(6,7)$.

We can conclude the following.

(I) There is no role for chemoradiotherapy in either the adjuvant or neoadjuvant setting.

(II) The standard of care for resectable pancreatic cancer is upfront resection with adjuvant multimodality chemotherapy.

(III) Neoadjuvant chemotherapy is appropriate for patients with borderline resectable or locally advanced pancreatic cancer.

In addition to the poor survival found in the prospective trial by Sohal et al. this study from Kizy et al. has shown that neoadjuvant therapy should not be used in patients with resectable pancreatic cancer, given the high level of nonresectability unless as part of a clinical trial (5).

\section{Acknowledgments}

Funding: None.

\section{Footnote}

Provenance and Peer Review: This article was commissioned by the editorial office of Hepatobiliary Surgery and Nutrition. The article did not undergo external peer review.

Conflicts of Interest: All authors have completed the ICMJE uniform disclosure form (available at https://hbsn. amegroups.com/article/view/10.21037/hbsn-21-131/coif). The authors have no conflicts of interest to declare.

Ethical Statement: The authors are accountable for all aspects of the work in ensuring that questions related to the accuracy or integrity of any part of the work are appropriately investigated and resolved.

Open Access Statement: This is an Open Access article 
distributed in accordance with the Creative Commons Attribution-NonCommercial-NoDerivs 4.0 International License (CC BY-NC-ND 4.0), which permits the noncommercial replication and distribution of the article with the strict proviso that no changes or edits are made and the original work is properly cited (including links to both the formal publication through the relevant DOI and the license). See: https://creativecommons.org/licenses/by-nc-nd/4.0/.

\section{References}

1. Kizy S, Altman AM, Wirth KM, et al. Systemic therapy without radiation may be appropriate as neoadjuvant therapy for localized pancreas cancer. Hepatobiliary Surg Nutr 2020;9:296-303.

2. Neoptolemos JP, Stocken DD, Friess H, et al. A randomized trial of chemoradiotherapy and chemotherapy after resection of pancreatic cancer. N Engl J Med 2004;350:1200-10.

3. Neoptolemos JP, Palmer DH, Ghaneh P, et al. Comparison of adjuvant gemcitabine and capecitabine with gemcitabine monotherapy in patients with resected pancreatic cancer

Cite this article as: Neoptolemos JP, Ghaneh P, Hackert T. Multimodality standard of care treatment of resectable and borderline resectable pancreatic cancer. HepatoBiliary Surg Nutr 2021;10(5):714-716. doi: 10.21037/hbsn-21-131
(ESPAC-4): a multicentre, open-label, randomised, phase 3 trial. Lancet 2017;389:1011-24.

4. Conroy T, Hammel P, Hebbar M, et al. FOLFIRINOX or Gemcitabine as Adjuvant Therapy for Pancreatic Cancer. N Engl J Med 2018;379:2395-406.

5. Sohal DPS, Duong M, Ahmad SA, et al. Efficacy of Perioperative Chemotherapy for Resectable Pancreatic Adenocarcinoma: A Phase 2 Randomized Clinical Trial. JAMA Oncol 2021;7:421-7.

6. Ghaneh P, Palmer DH, Cicconi S, et al. ESPAC-5F: Fourarm, prospective, multicenter, international randomized phase II trial of immediate surgery compared with neoadjuvant gemcitabine plus capecitabine (GEMCAP) or FOLFIRINOX or chemoradiotherapy (CRT) in patients with borderline resectable pancreatic cancer. J Clin Oncol 2020;38:abstr 4505.

7. Katz MHG, Shi Q, Meyers JP, et al. Alliance A021501: Preoperative mFOLFIRINOX or mFOLFIRINOX plus hypofractionated radiation therapy (RT) for borderline resectable (BR) adenocarcinoma of the pancreas. J Clin Oncol 2021:39:abstr 377. 